\title{
Du nouveau sur «Cannibal Jack »
}

Jean Guiart

\section{OpenEdition}

\section{Journals}

Édition électronique

URL : http://journals.openedition.org/jso/677

DOI : $10.4000 /$ jso. 677

ISSN : 1760-7256

Éditeur

Société des océanistes

Édition imprimée

Date de publication : 1 juin 2004

Pagination : 93-96

ISSN : 0300-953x

\section{Référence électronique}

Jean Guiart, « Du nouveau sur « Cannibal Jack » », Journal de la Société des Océanistes [En ligne],

118 | Année 2004-1, mis en ligne le 02 avril 2008, consulté le 01 mai 2019. URL : http://

journals.openedition.org/jso/677; DOI : 10.4000/jso.677 


\title{
MISCELLANÉES
}

\section{Du nouveau sur « Cannibal Jack »}

\author{
par Jean GUIART
}

\begin{abstract}
À propos de : William W. Emilsen (ed.), 1999, The Goldfields Journal of William Diaper (alias "Cannibal Jack»), 1851-1853 (Carlisle, Hesperian Press, 128 p., 30 gravures anciennes, 4 cartes).
\end{abstract}

Le révérend Emilsen a eu la bonne fortune de découvrir les manuscrits manquants de William Diaper, ou Diapea, ou John Jones, ou John Osborne, ou John Jackson, ou Fiji Jack, connu principalement sous le nom de "Cannibal Jack », cachés derrière une glace dans la bibliothèque de l'ancien United Theological College à Enfield, Nouvelle-Galles-du-Sud, Australie. J'ai eu le privilège, dont je le remercie, de contempler ce manuscrit, une écriture penchée régulière remplissant deux épais registres de l'époque, sous l'intitulé "Jack, the Cannibal-Killer», de la main de l'auteur lui-même. On cherchait ce manuscrit depuis un siècle. La découverte est d'importance.

On ne connaissait en effet jusqu'à présent que deux textes de William Diaper, l'un publié par le commandant britannique Erskine en annexe à son ouvrage classique sous le nom de John Jackson, l'autre recueilli à Maré et publié sous le nom de William Diapea par le missionnaire de la London Missionary Society James Hadfield. L'existence du manuscrit retrouvé avait été indiquée dans l'introduction par William Diaper au dernier texte publié par le révérend Hadfield. Les textes retrouvés se placent chronologiquement entre les deux manuscrits déjà connus. Le premier avait trait aux aventures, vraies ou fausses, de l'auteur aux îles Fidji et dans leur environnement insulaire et avait été écrit sur le navire de guerre HMs Havannah, à la demande du capitaine du navire, où l'auteur avait été engagé comme interprète pour ses capacités linguistiques évidentes. Le second traitait de la Polynésie occidentale, de Fidji et d'autres voyages, accessoirement de la Nouvelle-Calédonie.

Le manuscrit opportunément sorti de l'ombre a trait, d'une part, à la ruée vers l'or en Californie et surtout en Australie, et, d'autre part, dans un très grand détail, à son séjour en NouvelleCalédonie. Il sera le tout premier Européen à faire le tour de la Grande Terre, à pied ou en pirogue, et cela en 1853, juste avant la prise de possession française. Il est passé sans encombre à Pouébo et Balade, juste après la révolte des gens de Balade contre la présence des pères maristes et le meurtre d'un frère convers. $\mathrm{Ce}$ manuscrit a été rédigé à Pohnpei (Ponapé), aux îles Carolines orientales, à l'époque théoriquement espagnoles, où l'auteur s'était installé tout exprès pour rédiger, utilisant une encre faite par lui d'un mélange de charbon de bois broyé en poudre et d'urine, dans une situation où il ne trouvera pas exactement la tranquillité recherchée. Il espérait bien placer son manuscrit et en tirer un certain bénéfice financier. Il voulait aussi être connu comme un homme qui avait des aventures extraordinaires à relater.

L'ouvrage publié par le révérend Emilsen est fort bien présenté. L'appareil de notes n'est pas écrasant, mais il est largement suffisant, toujours pertinent, d'autant que l'éditeur a pris le soin de parcourir à nouveau, plus d'un siècle après, l'itinéraire suivi par Cannibal Jack d'un site de placer à un autre, tant en Nouvelle-Galles-du-Sud que dans le Victoria, de façon à retrouver si 
possible les descendants des personnes citées et vérifier leur existence au moins dans les archives locales. Il ressort de cet important travail de terrain que très peu d'erreurs de lieux ou de noms ont été commises par l'auteur du manuscrit. On aimerait que tous les historiens du Pacifique fassent preuve d'autant de scrupules intellectuels et de professionnalisme dans la présentation de leurs dossiers. L'école historique australienne a su se renouveler parce qu'elle a emprunté à l'ethnologie l'outil de l'enquête de terrain, s'ajoutant à la seule compulsion des archives (laquelle conduit à affirmer sans preuve, le contenu d'un document d'archives devant toujours être soigneusement vérifié sur le terrain). La qualité des illustrations bien choisies est tout à fait intéressante. Elles apportent des éléments d'information complémentaires sur la vie quotidienne des chercheurs d'or. L'image en couleurs de la couverture, une gravure d'époque, témoigne de l'antisémitisme régnant à l'époque dans le milieu des chercheurs d'or: des mineurs discutant sur le prix avec un trader acheteur d'or, sentiment que partageait l'auteur, cherchant en d'autres occasions à rouler un autre trader pratiquant le même métier, pour le sport.

Le texte traitant de la Nouvelle-Calédonie est en train d'être préparé pour l'édition. Le révérend Emilsen vient de se rendre en NouvelleCalédonie avec son épouse à cet effet. Je lui ai fourni tout ce que je pouvais comme moyens de déterminer quels étaient les personnes citées et les lieux de passage indiqués, du moins lorsqu'ils n'étaient pas évidents. De ce que j'ai vu de ce manuscrit, William Diaper est bien passé là où il le dit, et cette seule information sera précieuse à bien des points de vue.

Le problème posé dans chacun des textes de Cannibal Jack n'est pas la véracité des lieux ni celle des personnes citées, mais la réalité des événements relatés.

Sa capacité de résistance physique et de tenter des aventures, y compris en plein désert australien, aventures qu'aucun homme raisonnable n'aurait entreprises, surprend. Il marche sans fin comme s'il voulait éprouver sa capacité de poursuivre un effort au-delà de la limite satisfaisant la plupart de ses contemporains. Son tour de Nouvelle-Calédonie aurait effrayé tout autre. Il est d'ailleurs le seul à l'avoir tenté à l'époque. Personne n'a imaginé de rivaliser avec lui au cours ${\mathrm{du} \mathrm{XIX}^{\mathrm{e}}}^{\mathrm{e}}$ siècle, du moins avant que le contrôle administratif ne soit suffisamment établi, et de ce fait devenu rassurant. Il a beau parler de cannibales toujours à la recherche de chair humaine, personne n'a tenté de le mettre au four, ni de le tuer pendant son sommeil, ou à tout autre moment où il était véritablement sans défense, alors qu'au même moment une bande de chercheurs d'or disparaissait dans la haute vallée de Houailou. À voir comment les mêmes se comportaient en Australie, on imagine parfaitement qu'ils aient provoqué la volonté de les annihiler.

Un siècle et demi plus tard, je suis passé aux mêmes endroits, à une époque où il n'y avait guère de routes, soit dans une vieille jeep, soit surtout à cheval. J'ai une certaine idée des difficultés physiques surmontées par l'auteur des manuscrits et lui tire mon chapeau. C'était le temps de ces Canaques, grands marcheurs devant l'Éternel, que la poste coloniale utilisera comme porteurs de plis. On a toujours été plus en sécurité dans le pays kanak que dans les périmètres coloniaux que mon beau-père, $\mathrm{M}$. Jules Calimbre, partant de Nouméa, ne traversait à cheval qu'avec un revolver à la ceinture.

William Diaper avait de l'instruction, ayant suivi l'enseignement de la Latin Grammar School à Dedham, dans le comté d'Essex en Angleterre. Il a donc ce qu'on appelle des « lettres » et aussi de l'orthographe. Son esprit est encombré d'un certain nombre de modèles littéraires. Ses voyages, vérifiables et passionnants pour un chercheur, lui servent de cadre où placer des histoires, des yarns, dont il revendique la réalité quelle que soit leur crédibilité. Dans l'ouvrage publié, une partie de ces récits donne l'impression d'être vraisemblable, sinon vrai, et une autre, d'être les éléments d'une tradition orale d'histoires racontées sur les mines et à propos de toutes les ruées vers l'or au monde, lesquelles constituaient, au cours des premiers mois ou des premières années, des zones de non-droit, comme on l'exprime aujourd'hui. C'est toujours le cas, par exemple au Brésil.

En ce qui concerne la Nouvelle-Calédonie, et comme pour Fidji, Tonga ou Samoa, où Cannibal Jack a vécu, sa tactique habituelle qui apparaît crédible était de se concilier les bonnes grâces d'un chef local, puis d'essayer d'en tirer le maximum d'avantages, en particulier pour un commerce très opportuniste, en plus de sa profession d'armurier ambulant, la fourniture d'épouses temporaires. Sa loyauté était cependant vagabonde. Il aurait été le beachcomber classique, oublié de tous, s'il n'avait eu cette capacité d'écrire qui n'est pas donnée à tout le monde.

Au-delà, s'il fallait croire, non seulement aux scènes de cannibalisme dont il prétend avoir été témoin, aux meurtres qu'il a soi-disant perpétrés, se prétendant attaqué et menacé, résistant victorieusement tant à mains nues qu'avec le 
secours d'une arme à feu, mais aussi au nombre de femmes violées et en particulier de femmes mariées, viols dont il se reconnaît coupable, en Nouvelle-Calédonie du moins, paraissant considérer ce dernier point comme un des impondérables de la vie quotidienne dans le PacifiqueSud, on en aurait plein les mains de traductions pas toujours particulièrement originales des idées a priori dont les ouvrages de l'époque sont remplis. S'il avait commis, seul (il rechignait à faire partie d'une de ces bandes d'Européens jouant de leur puissance de feu conjointe et qui ont tant massacré, en particulier aux îles Fidji), le quart de toutes les actions dont il se vante, il aurait été proprement exécuté. Il raconte luimême, à chaque fois, par quels moyens il aurait réussi à échapper à une mise à mort programmée. Je pense, trois quarts de siècle plus tard, à Kafeat Cidopwaan ma Juat, le dirigeant militaire de l'insurrection de 1917 sur la côte est, étranglant quelques années auparavant à Hienghène l'Anglais Harkinson parce que ce dernier avait violé sa femme. Ce que Cannibal Jack raconte est la raison évidente pour laquelle ce manuscrit est resté caché dans la bibliothèque d'un séminaire de théologie. Ce n'était pas une lecture convenable. Il n'a heureusement pas été détruit.

Un des points faibles de l'auteur est de vouloir se présenter comme le sauveur de jeunes femmes vouées à un mariage non désiré, ou même à la mort, soit qu'elles fussent prisonnières de guerre et désignées pour être mises au four, soit qu'elles fussent veuves et qu'on ait voulu les sacrifier, ce qui l'oblige à fuir avec elles et à changer rapidement de lieu de résidence, quittant une île pour une autre ou s'échappant dans un autre archipel. En dehors d'un destin alimentaire douteux, j'aurais tendance à croire qu'une part au moins de ces histoires, romantiques à souhait à la mode de l'époque, ait été réelle. J'ai eu moi-même l'occasion, une fois, chez les Big Nambas, au Nord-Malekula, de sauver une très jeune veuve que le frère de son mari s'apprêtait à pendre. Je n'ai pas eu à m'en occuper plus avant. Dès le lendemain matin, elle avait mis deux jours de marche entre elle et son beau-frère. Pourquoi pas Cannibal Jack?

Les fuites successives de ce dernier d'un point à un autre constituent un thème récurrent de l'histoire de sa vie. Elles paraissent véridiques. Une fois au moins, la raison pourrait être cellelà. Cependant, chacun des manuscrits connus à ce jour contient deux histoires de ce genre, ce qui fait quatre en tout, mais dont deux semblent bien représenter les versions différentes de la même aventure féminine. Combien la Nouvelle-
Calédonie fournira-t-elle le lieu d'autres versions sur le même thème ? Aucun exemple de ce type n'a inspiré par contre le récit de ses aventures australiennes. Dans l'Australie de l'époque, le danger d'être lynché ou condamné à la pendaison explique probablement cette prudence vis-àvis des femmes déjà en mains ou des jeunes filles.

Ce type d'aventures, le preux chevalier sauvant sans réfléchir et au péril de sa vie une princesse en danger, fait partie à la fois de la tradition orale des pays celtiques et du romantisme apprécié par la société victorienne, qui compensait en partie ainsi la violence sous-jacente à son apparence de respectabilité. Les écrits de William Diaper s'inscrivent ainsi pour une part dans un ensemble de comportements individuels et collectifs qui comportent, entre autres, l'engouement pour la cause de l'indépendance de la Grèce sous l'égide du poète Byron et la charge de la Brigade légère au siège de Sébastopol. Une des histoires féminines racontées par Cannibal Jack pour Fidji a d'ailleurs été reprise dans un poème de Byron, qui la situera à Tahiti.

Le sort des femmes néo-calédoniennes violées par ce dernier ne le concerne par contre pas, alors qu'elles pouvaient être accusées de lui avoir cédé volontairement et, de ce fait, sanctionnées lourdement. Apparemment, Cannibal Jack n'a pas entendu parler des femmes adultères de Nouvelle-Calédonie châtiées par l'imposition sur le sexe d'une pierre rougie au feu. Il est concevable que ce soit là aussi une invention. Lorsque j'étais étudiant en stage au musée de l'Homme, les collections conservaient encore un "sexe de femme adultère" de NouvelleCalédonie. Le dit sexe, plutôt racorni, ne comportait aucune trace de brûlures. Curieusement, ni le manuscrit remis au commandant Erskine, ni celui donné au révérend Hadfield, ne contient l'aveu d'un viol du fait de l'auteur.

De même, seul le manuscrit remis au commandant Erskine nous apporte une description qui se veut fouillée de la société fidjienne traditionnelle et de son fonctionnement, description qui vise à émuler celle de James Mariner pour Tonga, ouvrage que Cannibal Jack avait lu dans la bibliothèque du bord, puisqu'il le cite dans le détail (il a servi comme interprète à bord de deux navires de guerre britanniques: les HMS Fly et Havannah). Il en ressort l'artificialité de la prééminence, construite sur le commerce d'armes à feu, de la lignée des Cakobau et l'importance première des chefferies de Cakaudrove et de Macuata à Vanua Levu. Le rôle de Tui Nayau est bien marqué, de même qu'apparaît déjà le nom de Ratu Mara. Tous ces gens, ou plutôt leurs descendants, surgiront du passé à l'occasion des 
troubles qui suivront les putschs militaires destinés à éviter l'installation d'une suprématie politique hindoue dans ce pays.

William Diaper décrit l'installation d'équipes de charpentiers venus de Tonga et y repartant avec une pirogue de haute mer à deux coques, toute neuve, construite avec des troncs de pins kaoris fidjiens, pour un voyage de retour plus difficile et plus aléatoire que le voyage aller placé sous les alizés. Toute cette description est exacte en beaucoup de points, comportant même des précisions ethnographiques originales, si du moins on met de côté les histoires de massacres et de cannibalisme où il en rajoute constamment. Ce côté devait arranger les nombreux missionnaires méthodistes qu'il a fréquentés et auquel il doit peut-être une partie de ses informations (ses descriptions précises et détaillées de la façon d'être de certains missionnaires qui l'ont aidé, ou même qui l'ont employé, sont intéressantes, sinon même divertissantes). Certaines descriptions de prêcheurs protestants en rivalité dans l'outback australien valent leur pesant d'or.

Les noms de lieux et de personnages, si importants par leurs liens mutuels, sont donnés convenablement, y compris avec les nuances qui s'imposent dans certains cas, nuances qu'il a su déceler ou qui lui ont été enseignées. L'un dans l'autre, les capacités intellectuelles de l'auteur apparaissent sous un jour très favorable dans certains récits qui mériteraient de devenir classiques. La qualité de l'écriture et de l'information est bien supérieure, ici pour Fidji, à bien des ouvrages de la littérature européenne locale en Nouvelle-Calédonie où, même aujourd'hui, les Kanaks décrits sont toujours des Kanaks imaginaires.

On notera que le thème des corps de prisonniers utilisés vivants pour servir de rouleaux pour remonter à terre les grandes pirogues de haute mer, thème que Teuira Henry développe en ce qui concerne les soi-disant cérémonies attirant toute la Polynésie qui auraient eu lieu au marae Taputapuatea de Raiatea tous les vingt ans, est donné aussi en bien plus grand détail par Cannibal Jack pour l'île fidjienne de Mbengga, sans en proposer d'autre explication que la visite de personnages de haut rang venant de la vallée de Rewa et sans qu'il prétende qu'il s'agisse là d'une tradition commune à l'ensemble de l'archipel. On a l'impression qu'il case là un récit qu'il voulait placer à toute force.

On notera enfin qu'une partie de la vie de Cannibal Jack reste floue, celle où il réussit à échapper à Hienghène aux troupes françaises et ainsi à éviter d'être fusillé contre un rocher en bas de la gendarmerie comme trois autres traders anglais. Il gagnera Samoa dans une baleinière non pontée, ce qui est un exploit, baleinière qu'il aurait volée avec deux autres compagnons. Au cours de la période qui suivra, il partait se cacher dans la montagne chaque fois qu'un navire de guerre français entrait en rade d'Apia.

William Diaper est mort à Maré le 4 mars 1891. Le révérend Hadfield venait de constater, à sa grande surprise, qu'il parlait couramment le nengone, la langue de l'île de Maré. On dit qu'il y aurait séjourné très jeune, arrivé là sur son tout premier bateau, où il était mousse, le Joshua Caroll. Le révérend Hadfield raconte que lorsque le jeune homme était venu déjeuner chez le grand chef de Wetr, ce dernier envoya auparavant son exécuteur assommer un passant dont ils puissent manger la chair, à laquelle William Diaper aurait refusé de toucher. L'histoire est certainement controuvée. Cette forme de cannibalisme alimentaire à la discrétion des chefs voulant satisfaire une envie est justement celle que racontaient les missionnaires de presque partout (excepté aux îles Samoa) à l'époque, aux fins de justifier ce qu'ils présentaient comme leur apostolat et qui pouvait l'être ou pas.

\section{RÉFÉRENCES}

DiapeA, William, 1928. Cannibal Jack, The True Autobiography of a White Man in the South Seas, Londres, Faber et Gwyer, 236 p.

ERskine, John Elphinstone, 1853, Journal of a Cruise Among the Islands of the Western Pacific... in Her Majesty's Ship Havannah, Londres, Dawsons (cf. « Jackson's Narrative », pp. 412-477).

Legge, Cristopher, 1966, «William Diaper, A Biographical Sketch », Journal of Pacific History 1, Canberra, pp. 70-90. 